\title{
A STUDY ON THE DIRECT CONVERSION OF METHANE TO METHANOL
}

\author{
MORTEZA SOHRABI \# \& LEILA VAFAJOO * \\ (\#) Amirkabir University of Technology, Department of Chemical Engineering, Petrochemical Centre of Excellence, Tehran 15914, Iran, and I.R. Academy of \\ Sciences, Tehran 19717, Iran \\ (*) Islamic Azad University, Tehran South Branch, Graduate School of Engineering, Chemical Engineering Faculty, Mollasadra Ave., Tehran, Iran. \\ (Received: July 22, 2008 - Accepted: March 7, 2009)
}

\begin{abstract}
The selective oxidation of methane to methanol has been investigated in a packed bed reactor applying $\mathrm{V} 2 \mathrm{O} 5 / \mathrm{SiO} 2$ as the reaction catalyst. The effects of certain pertinent parameters of the system such as temperature, pressure and gas hourly space velocity (GHSV) on the conversion of methane and selectivity of the products have been investigated. It has been observed that by increasing the conversion of methane, the selectivity of both $\mathrm{CO}$ and formaldehyde is enhanced, while, that of methanol and $\mathrm{CO} 2$ is reduced. This could be due to the formation of certain by products such as formaldehyde as the result of further oxidation of methanol. Temperature was found to have the highest effect on the rate of reaction. A simple kinetic model has been devised for this process and the kinetic parameters have been determined as functions of temperature. The data predicted from the model have been correlated with those determined experimentally. The mean absolute deviation between the two sets of data was ca $7 \%$.
\end{abstract}

Keywords: selective oxidation, methanol, catalytic reaction, kinetic model, methane to methanol

\section{INTRODUCTION}

The difficulties associated with the transportation of natural gas from production fields to consumption ports have persuaded the industrial and scientific institutions to develop certain methods for conversion of natural gas to certain value added liquid chemicals. Presently, the major part of natural gas is burned to provide thermal energy, and only a minor portion of the latter is converted to synthesis gas by steam reforming, which in turn is converted to various products such as methanol and formaldehyde.

The two-step process for conversion of natural gas to methanol via production of synthesis gas is a cost bearing method, mainly due to the limitations imposed by the reaction equilibrium and low heat efficiency ${ }^{1}$. Efforts are, therefore, being made to devise economical processes for direct conversion of methane (the major constituent of natural gas) to methanol and formaldehyde.

With nearly 26 million tones annual global consumption, methanol has gained a special rank among the consumable chemicals ${ }^{2}$. More than one-third of methanol produced is converted to formaldehyde. It is also utilized for production of acetic acid and some additives for high octane number gasoline ${ }^{3}$. Further applications of methanol are in MTO (methane to olefins) and MTG (methane to gasoline) processes ${ }^{4}$. It may be predicted, therefore, that by increasing demand for methanol in future, economical production of this chemical will be increasingly propounded ${ }^{5}$.

Some experimental studies have been conducted to direct oxidation of methane to methanol and formaldehyde. These studies can be classified into two major areas, i.e. partial oxidation in homogeneous media and that in heterogeneous systems. In a study by yarlagada et $\mathrm{al}^{5}$ methanol was produced in a homogeneous gas phase reaction using a mixture of $5 \%$ oxygen and $95 \%$ methane. A tubular reactor was applied at 65 bar pressure and $450{ }^{\circ} \mathrm{C}$. Conversion of methane was $6.5-10 \%$. Anthony and Chen ${ }^{6}$ studied the noncatalytic partial oxidation of methane to methanol. They presented a mechanism consisted of a number of free radicals elementary reactions and concluded that with $2.3-4.4 \%$ oxygen in feed and 50 bar pressure the selectivity of methanol did not exceed $55 \%$. In a further study by these authors ${ }^{7}$, the homogeneous gas phase oxidation of methane to methanol was carried out in a tubular reactor. They observed that by increasing the temperature or oxygen concentration in feed, selectivity of methanol was reduced. Casey et al ${ }^{8}$ conducted a study on the direct non-catalytic oxidation of methane to methanol and formaldehyde and concluded that in the range of $30-60$ bar pressure and within $300-500^{\circ} \mathrm{C}$, the yield of methanol was $1.8 \%$. In a paper published by Arutyunov and co-workers ${ }^{9}$ the partial oxidation of natural gas to methanol, applying high pressures, was reported. They demonstrated that pressures in excess of 180 bar, reduced the yield of methanol. Lodeng et al ${ }^{10}$ investigated the oxidation of methane to methanol in a homogeneous system and observed that with 2.6$4.4 \%$ oxygen in feed, $40-60$ bar pressure and $450-550^{\circ} \mathrm{C}$, methane conversion was $1.2-2.6 \%$. Hunter et al ${ }^{11}$ applied a tubular reactor packed with $\mathrm{SnO}_{2}$ as the reaction catalyst. The operating conditions were 30 bar pressure, $247^{\circ} \mathrm{C}$ and the mean residence time of $23 \mathrm{~s}$. The selectivity of methanol and conversion of methane were $87 \%$ and $4 \%$, respectively. The catalyst was deactivated rapidly. Chellappa and Viswanath ${ }^{12}$ used a feed with the oxygen to methane molar ratio of $1 / 16$. The catalyst was supported ferric molybdate and the operating temperature and pressure were 34 bar and $743 \mathrm{~K}$, respectively. Methanol selectivity was $84 \%$ and methane conversion was $5.67 \%$. Walker and coworkers ${ }^{13}$ applied a fixed bed reactor at 54 bar pressure, using various metal oxides catalysts such as $\mathbf{g}$-alumina, $\mathrm{MgO}, \mathrm{MoO}_{3}$ and $\mathrm{SnO}_{2}$. Feed contained $5 \%$ oxygen. The methanol selectivity was low and did not exceed $35 \%$; while the methane conversion was in the range of 3-4.5\%. Chen and Wilcox ${ }^{14,15}$ investigated the effect of water vapor on the reactor's performance. The operating temperature was $460^{\circ} \mathrm{C}$ and the reaction catalysts were $\mathrm{V}_{2} \mathrm{O}_{5}$ and $\mathrm{V}_{2} \mathrm{O}_{5} / \mathrm{SiO}_{2}$. In a part of their experiments, mixtures of $\mathrm{O}_{2}$ and $\mathrm{N}_{2} \mathrm{O}$ were used as the oxidizing agent. The mean residence time was $1.4 \mathrm{~s}$ and the conversion of methane was in the range of $2-15 \%$. In a study performed by Chun et al ${ }^{16}$, partial oxidation of methane was achieved using $\mathrm{V}_{2} \mathrm{O}_{5} / \mathrm{SiO}_{2}$ as the catalyst. The operating parameters were 50 bar pressure, $640-750 \mathrm{~K}$ temperature and $2-5 \mathrm{~s}$ residence times. They observed that increase in methane conversion was accompanied with the drop in methanol selectivity. In a paper published by Tabata and co-workers ${ }^{17}$, the effects of certain radical generative additives such as $\mathrm{NO}$ on the conversion of methane and prevention of deep oxidation of $\mathrm{C}_{1}$ - oxygenates were discussed. Application of nitrogen oxides $\left(\mathrm{NO}_{x}\right)$ in promotion of gas phase reaction was also considered. The rate determining step of the selective oxidation of methane was assumed to be the abstraction of the first hydrogen from $\mathrm{CH}_{\text {. }}$. However, the high dissociation energy associated with such a process could lead to sequential oxidation of $\mathrm{C}_{1}$-oxygenates to carbon monoxide and carbon dioxide. The authors finally concluded that the precise mechanisms for the combined gas phase and solid catalytic reactions have not yet been fully clarified. In a more recent paper published by Tabata et al ${ }^{18}$, the role of germanium ion incorporated into tin oxide in the selectivity of products formed in partial oxidation of methane was investigated in detail. They applied $\mathrm{Sn}_{1-\mathrm{x}} \mathrm{Ge}_{\mathrm{x}} \mathrm{O}_{2}(\mathrm{x}=0-0.5)$ as the reaction catalyst in presence of NO. The main product among $C_{1}$-oxygenates was formaldehyde. The authors assumed separate routes for homogeneous gas phase and heterogeneous catalytic reactions for formaldehyde formation. Iglesia and co-workers ${ }^{19}$, examined a detailed kinetic network for $\mathrm{CH}_{4}-\mathrm{O}_{2}-\mathrm{NO}_{x}$ reactions system in order to identify the elementary steps that promote the effects of $\mathrm{NO}_{\mathrm{x}}$ on methane oxidation rates and on transformation of $\mathrm{NO}_{x}$ to non reactive $\mathrm{N}$-compounds. The authors demonstrated that enhancement in methane oxidation rates due to the presence of $\mathrm{NO}_{\mathrm{x}}$ could be the result of reaction between $\mathrm{NO}_{2}$ and $\mathrm{CH}_{4}$ during an initial induction period. Decrease in selectivity of $\mathrm{C}_{1}$-oxygenates with increasing $\mathrm{CH}_{4}$ conversion was related to the weaker $\mathrm{C}-\mathrm{H}$ bonds in these products relative to that in $\mathrm{CH}_{4}$. This could explain the fast sequential oxidation of $\mathrm{C}_{1}$-oxygenates to $\mathrm{CO}$ and $\mathrm{CO}_{2}$. They also claimed that $\mathrm{NO}_{\mathrm{x}}$ molecules induce certain effective elementary steps for formation of $\mathrm{CH}_{3} \mathrm{O}$ radicals and conversion of the latter to formaldehyde. In addition, increase in $\mathrm{C}_{1}$-oxygenates yields with increasing $\mathrm{O}_{2}$ partial pressure could be due to the inhibitive effect of oxygen on conversion of $\mathrm{NO}_{\mathrm{x}}$ to some less reactive $\mathrm{N}$-compounds.

The major objective of the present study was to devise a kinetic model, based on active sites concept for the catalytic partial oxidation of methane. The model had to be simple, yet pertinent for the purpose of reactor design. In addition, it was aimed to investigate further, the effects of certain operating parameters such as temperature, pressure and gas hourly space velocity (GHSV) on conversion of methane to methanol and selectivity of products. 


\section{EXPERIMENTAL}

\section{Process parameters}

The pertinent process parameters were as follows,

catalyst's bed temperature: $450-500^{\circ} \mathrm{C}$; methane flow rate: $20-250 \mathrm{~cm}^{3}$ min; oxygen flow rate: $1.2-5 \mathrm{~cm}^{3} / \mathrm{min}$; nitrogen flow rate: $0-5 \mathrm{~cm}^{3} / \mathrm{min}$; weight of catalyst/molar flow rate of methane $\left(\mathrm{W} / \mathrm{F}_{\mathrm{o}}\right): 5.9-29.5$ (g catalyst $) /(\mathrm{g}$ mol $\left.\mathrm{CH}_{4}\right)(\mathrm{h})$; weight of catalyst: $1-5 \mathrm{~g}$; GHSV: $300-1200 \mathrm{~h}^{-1}$; porosity of the catalyst bed: $40 \%$; porosity of the catalyst grain: $45 \%$; average diameter of the catalyst grain: $0.2-0.8 \mathrm{~mm}$; length of the catalyst bed: $1-6 \mathrm{~cm}$.

\section{Materials}

All chemicals applied in this study were of analytical grade.

\section{Catalyst}

The catalyst used in the present study was a commercial specimen, Monsanto type 210. The latter were ring shape pellets with the average dimensions of $8 \mathrm{~mm}$ in diameter, $10 \mathrm{~mm}$ height and $2 \mathrm{~mm}$ thickness. Some of the physicochemical properties of the catalyst are given in Tables 1 and 2.

The catalysts pellets were crushed into pieces having $0.2-0.8 \mathrm{~mm}$ size, prior to use.

Table 1. Some of the physical characteristics of the catalyst.

\begin{tabular}{|c|c|c|c|}
\hline Properties & Grain form & Pellet form & Unit \\
\hline Surface area & 1.72 & 1.40 & $\mathrm{~m}^{2} / \mathrm{g}$ \\
\hline Pore volume & $5.29 \times 10^{-4}$ & $4.1 \times 10^{-4}$ & $\mathrm{~cm}^{3} / \mathrm{g}$ \\
\hline Average pore diameter & 34.6 & 35.9 & ${ }^{\circ} \mathrm{A}$ \\
\hline
\end{tabular}

Table 2. Chemical composition of the catalyst.

\begin{tabular}{|c|c|}
\hline Components & Weight\% \\
\hline $\mathrm{SiO}_{2}$ & 59.2 \\
\hline $\mathrm{V}_{2} \mathrm{O}_{5}$ & 5.3 \\
\hline $\mathrm{SO}_{3}$ & 18 \\
\hline $\mathrm{K}_{2} \mathrm{O}_{5}$ & 8.5 \\
\hline $\begin{array}{c}\text { Other constituents such as } \mathrm{Fe}_{2} \mathrm{O}_{3}, \mathrm{MgO}, \mathrm{Cs}_{2} \mathrm{O}, \mathrm{MoO}_{3}, \\
\mathrm{Na}_{2} \mathrm{O}, \mathrm{Al}_{2} \mathrm{O}_{3}\end{array}$ & 9 \\
\hline
\end{tabular}

\section{Experimental set up}

The experimental rig consisted of a tubular reactor made of stainless steel type $316,15 \mathrm{~mm}$ internal diameter and $160 \mathrm{~mm}$ length, placed inside an electric oven equipped with an automatic temperature regulator. The gaseous reactants $\left(\mathrm{N}_{2}, \mathrm{O}_{2}\right.$, and $\left.\mathrm{CH}_{4}\right)$ entered the reactor via three mass flow controllers (Brooks, model 0154). The depth of the catalyst bed was in the range of 10-60 mm. The bed temperature was measured by a thermocouple housed in the latter. The outlet gas, after passing a condenser and an adsorber, directed to a GC on line apparatus (Shimadzu, model 14B). The adsorber was filled with silica gel in order to adsorb the water vapor present in the gas mixtures. A schematic diagram of the set up is shown in Figure 1.

Two successive columns were used to analyze the reactor exit gas components. Both columns were $1.8 \mathrm{~m} \times 3 \mathrm{~mm}$ tubes made of stainless steel. The first was packed with Porapak S of 80-100 mesh and used for the determination of methanol and formaldehyde, while the second column was filled with molecular sieve $5 \mathrm{~A}$. The columns temperatures were 180 and $50^{\circ} \mathrm{C}$ respectively and thermal conductivity detector was employed. Helium was applied as the carrier gas at the constant flow rate of $30 \mathrm{~cm}^{3} / \mathrm{min}$.

\section{RESULT AND DISCUSSION}

Kinetic model

Based on Langmuir-Hinshlewood formulation, a kinetic scheme was derived for the direct conversion of methane to methanol as follows,

$$
\begin{aligned}
& \mathrm{CH}_{4}(\mathrm{~g})+s \underset{k_{1}^{\prime}}{\stackrel{k_{1}}{\rightleftarrows}} \mathrm{CH}_{4} \cdot s \\
& \mathrm{O}_{2}(\mathrm{~g})+2 s \stackrel{\mathrm{k}_{2}}{\underset{k_{2}^{\prime}}{\longrightarrow}} 2 \mathrm{O} \cdot s \\
& \mathrm{CH}_{4} \cdot \mathrm{s}+\mathrm{O} \cdot \mathrm{s} \stackrel{k_{3}}{\longrightarrow} \mathrm{CH}_{3} \mathrm{OH}(\mathrm{g})+2 \mathrm{~s}
\end{aligned}
$$

where, $s$, stands for an active site.

Reactions 1 and 2 were assumed to be in equilibrium, while the overall rate is governed by reaction 3 . Hence,

$$
\begin{aligned}
& r_{1}=k_{1} P_{C H_{4}}\left(1-\theta_{1}-\theta_{2}\right)-k_{1}^{\prime} \theta_{1}=0 \\
& r_{2}=k_{2} P_{O_{2}}\left(1-\theta_{1}-\theta_{2}\right)^{2}-k_{2}^{\prime} \theta_{2}^{2}=0 \\
& r_{3}=k_{3} \theta_{1} \theta_{2}
\end{aligned}
$$

where, $s$, stands for an active site.

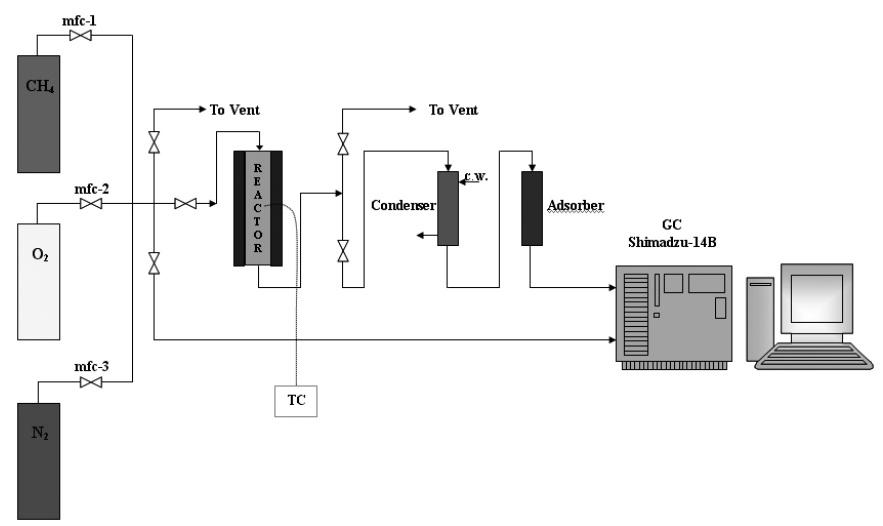

Figure 1. Schematic diagram of the experimental rig.

Reactions 1 and 2 were assumed to be in equilibrium, while the overall rate is governed by reaction 3 . Hence,

$$
\begin{aligned}
& r_{1}=k_{1} P_{C H_{4}}\left(1-\theta_{1}-\theta_{2}\right)-k_{1}^{\prime} \theta_{1}=0 \\
& r_{2}=k_{2} P_{O_{2}}\left(1-\theta_{1}-\theta_{2}\right)^{2}-k_{2}^{\prime} \theta_{2}^{2}=0 \\
& r_{3}=k_{3} \theta_{1} \theta_{2}
\end{aligned}
$$

where, $P_{\mathrm{CH}_{4}}$ and $P_{\mathrm{O}_{2}}$ are the partial pressures of methane and oxygen, respectively, $k_{1}$ and $k_{2}$, are the adsorption coefficients for methane and oxygen, respectively, $k_{3}$, is the rate coefficient for the adsorbed species, $k_{1}^{\prime}$ and $k_{2}^{\prime}$ are the desorption coefficients for the adsorbed methane and oxygen, respectively, $\theta_{1}$ and $\theta_{2}$, are the catalyst's active sites occupied by methane and oxygen, respectively.

By some mathematical manipulation, the following expression for the rate of methanol formation is obtained.

$$
\begin{gathered}
\text { Rate }=\frac{k_{3} K_{1} \sqrt{K_{2}} P_{C H_{4}} P_{O_{2}}^{0.5}}{\left(1+\sqrt{K_{2}} P_{O_{2}}^{0.5}+K_{1} P_{C H_{4}}\right)^{2}} \\
\text { where, } K_{1}=\frac{k_{1}}{k_{1}^{\prime}} \text { and } K_{2}=\frac{k_{2}}{k_{2}^{\prime}}
\end{gathered}
$$

where, and (sorption equilibrium constants for methane and oxygen, respectively).

The above model may be simplified by considering that under experimental conditions employed in the present study (Table 1 ) the methane partial pressure is 17-50 times greater than that of oxygen. Therefore, the term $\sqrt{K_{2}} P_{O_{2}}^{0.5}$ may 
be dropped from the denominator of equation 4. The rate expression is thus, reduced to the following relation,

$$
\text { Rate }=\frac{k P_{\mathrm{CH}_{4}} P_{\mathrm{O}_{2}}^{0 \mathrm{~S}}}{\left(1+K P_{\mathrm{CH}}\right)^{2}}
$$

where, $k=k_{3} K_{1} \sqrt{K_{2}}$ and $K=K_{1}$

Assuming the plug flow of fluid in the packed bed reactor, the following relation may be applied to determine the rate of reaction.

$$
\frac{W}{F_{0}}=\int_{0}^{x} \frac{d X}{\text { Rate }}
$$

Where, $W$, is the weight of catalyst; $F_{0}$, is the reactant's molar flow rate and $X$, is the conversion.

However, as the methane conversion in all experimental run was low (less than $8 \%$ ) an "average rate" could be defined as follows,

$$
(\text { Rate })_{\text {derage }}=\frac{F_{0}\left(X_{\text {out }}-X_{\text {in }}\right)}{W}
$$

Values for $k$ and $K$, as functions of temperature, were determined from the experimental data, applying non-linear least square method.

$$
\begin{array}{lll}
k=1.21 \times 10^{7} e^{-1540 / T} & \left(g_{\text {molCH}} \mathrm{OH}\right) /(\text { gcatalyst })(h)(\text { bar })^{15} & (T=K) \\
K=2.10 \times 10^{-5} e^{6800 / T} & (\text { bar })^{-1} & (T=K)
\end{array}
$$

In order to test the validity of the kinetic model, a comparison has been made between the rate data predicted from the model (equation 5) and those determined experimentally, applying equation 7 . The predicted data have been plotted against the experimental results in Figure 2. The mean absolute deviation between the two sets of data was found to be less than $7 \%$.

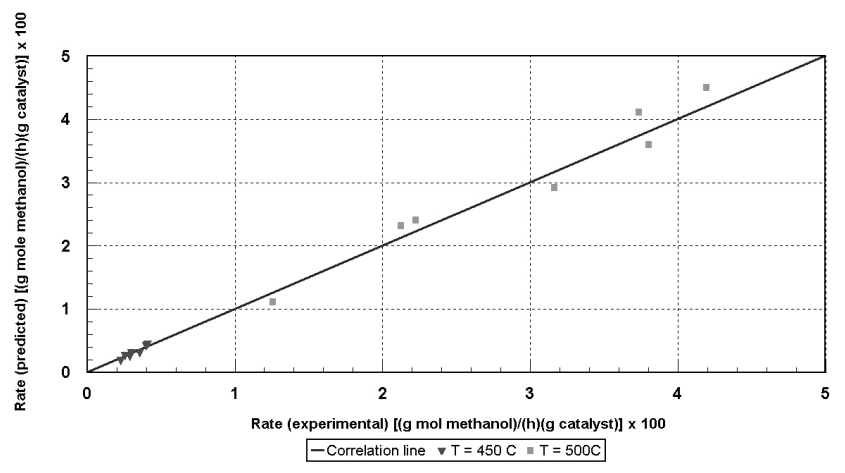

Figure 2. Comparison between the experimental rate data and those predicted from equation 5 .

Effect of some pertinent process parameters on conversion of methane and products selectivity

The effects of certain operating variables on the behavior of the process were considered, the results of which are presented in Figures 3-7 and in the following sub-sections.

Effect of temperature

In a previous study ${ }^{20}$ on direct conversion of methane to methanol, the optimum operating parameters have been determined using Taguchi experimental design method. In such an investigation the highest selectivity of methanol was observed with a feed composition of $5 \%$ oxygen and $95 \%$ methane. This feed composition was applied throughout the present study. The experimental results obtained under steady state conditions, indicated that increase in temperature from 450 to $500{ }^{\circ} \mathrm{C}$ at three different GHSV $(400,720$ and $1400 \mathrm{~h}^{-1}$ ) enhanced conversion of methane and lowered the selectivity of methanol. The results are shown in Figures 3 and 4 . At the same time, selectivity of $\mathrm{CO}$, Formaldehyde and $\mathrm{CO}_{2}$ were all increased. As it is apparent from Figure 2 , the effect of temperature on the rate of reaction is more pronounced than that of GHSV. For instance, a $50^{\circ} \mathrm{C}$ increase in temperature, promoted the methane conversion by five folds, whereas, decreasing the GHSV from 1400 to $400 \mathrm{~h}^{-1}$ only tripled such a conversion.

In order to compare the experimental data presented by some authors, with those determined in the present study, the properties of the catalysts applied by the latter authors should be known. However, such pieces of information are not always available in the literature and as such; the precise comparison is not possible. Nevertheless, a general agreement among the data obtained in this study and those from diverse reaction systems has been observed.

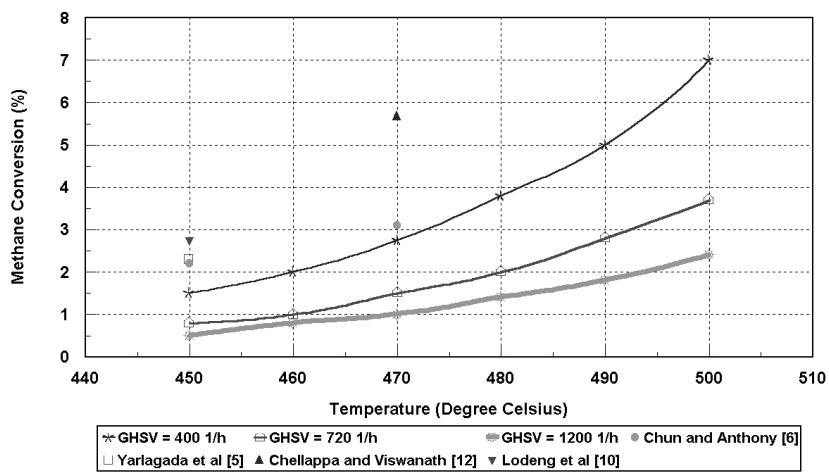

Figure 3. Effect of temperature on conversion of methane

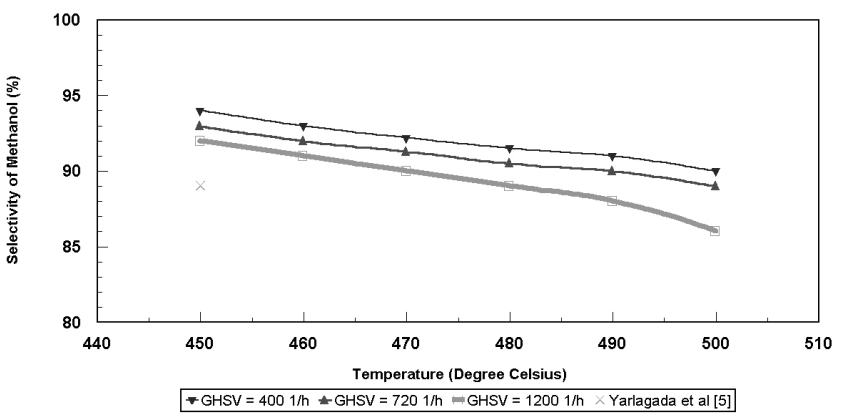

Figure 4. Effect of temperature on the selectivity of methanol

With regard to the present data, it seems that the rates of formation of some by-products are more temperature sensitive than that of methanol. Thus, although increase in temperature promotes the methane conversion, nevertheless, the methanol selectivity is reduced.

\section{Effect of pressure}

The results indicated that by increase in pressure of the system at a fixed temperature of $500{ }^{\circ} \mathrm{C}$ the selectivity of methanol was remained almost unchanged (Figure 5) while that of $\mathrm{CO}$ was enhanced. However, selectivity of $\mathrm{CO}_{2}$ and conversion of methane were only slightly affected by the pressure change. 


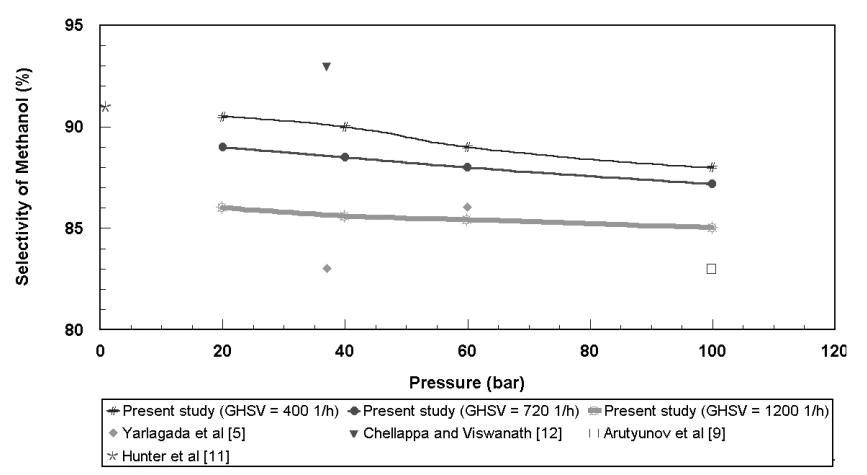

Figure 5. Effect of pressure on the selectivity of methanol

(Temperature $=500^{\circ} \mathrm{C}$ )

\section{Effect of feed flow rate}

The results indicated that increase in volumetric flow rate of feed at two different reaction temperatures $\left(450\right.$ and $\left.500^{\circ} \mathrm{C}\right)$ reduced the conversion of methane. This is due to the decrease in residence time of reactants within the reactor. In addition, it was noted that the selectivity of methanol was increased, while that of $\mathrm{CO}_{2}$ remained almost unchanged (Figure 6). In Figure7, the decrease in selectivity of methanol with increase in conversion of methane is shown. Reduction of methanol selectivity with increase in methane conversion could be the result of formation of certain by products such as formaldehyde from further oxidation of methanol with oxygen.

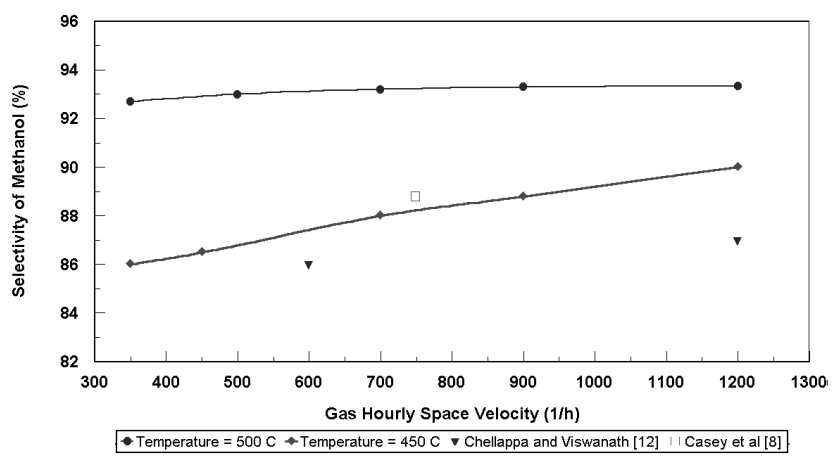

Figure 6. Effect of gas hourly space velocity (GHSV) on methanol selectivity.

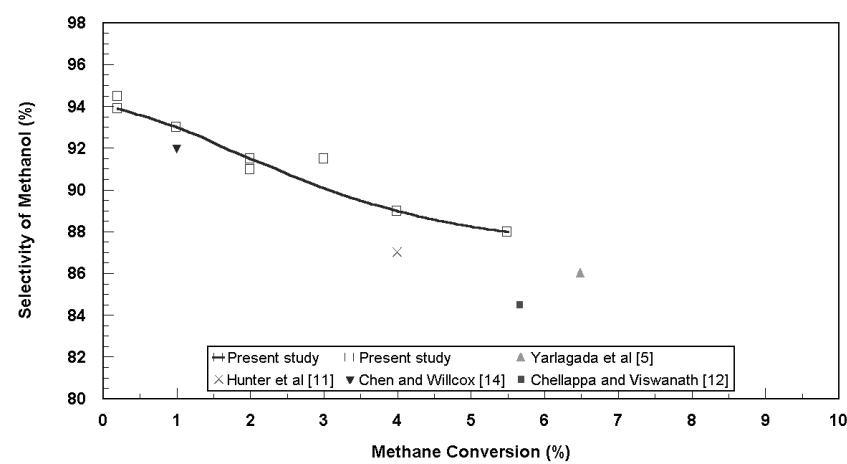

Figure 7. Relation between methanol selectivity and conversion of methane

Effect of catalyst pellet size

The effect of pellet size on conversion of methane is shown in Figureure 8. Such an observation may be explained by the catalyst's effectiveness factor defined as,

$$
\eta=\frac{\text { (rate of reaction affected by pore diffusion) }}{\text { (rate of reaction at the catalyst's external surface) }}
$$

In case of small particle size, neither external nor internal diffusion exists. Hence, the effectiveness factor tends to unity and higher conversions are expected. However, by increasing the particle size, resistance to pore diffusion is gradually built up and the $\eta$ values are dropped, causing a decrease in the rate of reaction and hence, conversion of methane.

It was found that the pellets having diameters less than $3 \mathrm{~mm}$, demonstrated an effectiveness factor close to unity, whereas, in case of pellets larger than 4 $\mathrm{mm}$ in size, the diffusional resistances affected the overall rate.

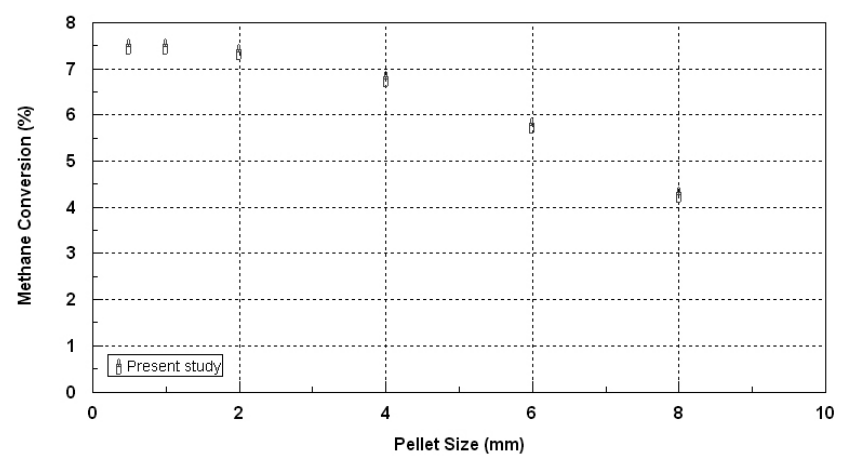

Operating Conditions: Temperature $=500^{\circ} \mathrm{C}$; Pressure $=100$ bar; Methane flow rate $=20 \mathrm{~cm}^{3} / \mathrm{min}$; Oxygen flow rate $=1.2 \mathrm{~cm}^{3} / \mathrm{min} ; \mathrm{GHSV}=300 \mathrm{~h}^{-1}$

Figure 8. Effect of catalyst pellet size on conversion of methane

\section{CONCLUSION}

In the present study, the direct conversion of methane to methanol and the factors affecting this process has been studied in a continuous catalytic packed bed reactor. In addition, a kinetic model has been devised for such a process based on Langmuir-Hinshlewood dual site formulation. The data calculated from the model have been compared with those determined experimentally. The absolute deviation between the two sets of data was ca $7 \%$.

Increase in temperature promotes the rates of by-products formation (presumably due to the higher activation energies of the side reactions) and hence, lowers the methanol selectivity. Changing the pressure of the reaction system has no appreciable effect on the selectivity of methanol.

In general, conversion of methane to methanol in a single pass of the reactant gas over the bed of catalyst is rather low. However, this may be largely improved by providing a recycle of the reaction products from the outlet to the entrance of the reactor. Such a procedure is commonly applied in the conventional production of methanol from synthesis gas.

\section{REFERENCES}

1. A.S. Chellappa, S. Fuangfoo, D. S.Viswanath, Ind. Eng. Chem. Res., 36, 1401 (1997).

2. J. R.Crocco, Hydrocarbon Processing, 1, 21 (2000).

3. D. W. Rytz, A. Baiker, Ind. Eng. Chem. Res., 30, 2287 (1991).

4. G. C.Chinchen, P. J. Denny, J. R. Jennings, M. S. Spencer, K. Waugh, Appl. Catal., 36, 1 (1988).

5. P. S. Yaralgadda, , A. Morton, N. R. Hunter,H. D. Gesser, Ind. Eng. Chem. Res., 27, 252 (1988).

6. J. W. Chun, R. G. Anthony, Ind. Eng. Chem. Res., 32, 788 (1993).

7. J. W. Chun, R. G. Anthony, Ind. Eng. Chem. Res., 32, 796 (1993).

8. Ph. S. Casey, T. McAllister, K. Foger, .Ind. Eng. Chem. Res., 33, 1120 (1994).

9. V. S. Arutyunov, , V. Y. Basevich, and V. I. Vedeneev, Ind. Eng. Chem. Res., 34, 4238 (1995).

10. R. Lodeng, O. A. Lindvag, P. Soraker, P. T. Roterud, O. T. Onsager, Ind. Eng. Chem. Res., 34, 1044 (1995).

11. N. R. Hunter, H. D. Gesser, L. A. Morton, P. S. Yarlaggada,. Appl. Catal, $\mathbf{5 7 ,} 45(1990)$

12. A. S. Chellappa, D. S. Viswanath, Ind. Eng. Chem. Res., 34, 1933 (1995).

13. G. S. Walker, J. A. Lapszewicz, G. A. Foulds, Catalysis Today, 21, 519 (1994). 
14. Sh. Y. Chen, D. Willcox, Ind. Eng. Chem. Res., 32, 584 (1993).

15. Sh. Y. Chen, D. Willcox, Ind. Eng. Chem. Res., 33, 832 (1994).

16. J. W. Chun, R. G. Anthony, Ind. Eng. Chem. Res., 32, 259 (1993).

17. K. Tabata, , Y. Teng, T. Takemoto, E. Suzuki, M.A. Pena, J.L.G. Fierro, Catal. Rev. Sci. Eng. 44, 1 (2002).
18. K. Tabata, T. Kawabe, Y. Yamaguchi, E. Suzuki, T. Yashima, J. Catal., 231, 430 (2005).

19. J. M. Zalc, W. H. Green, E.Iglesia, Ind. Eng. Chem. Res., 45 (8), 2677 (2006).

20. L. Vafajoo, PhD thesis, Amirkabir University of Technology, Tehran, Iran, 2002. 